\title{
A simple proof of a strong comparison principle for semicontinuous viscosity solutions of the prescribed mean curvature equation *
}

\author{
Masaki OHNumA ${ }^{\dagger}$ and Shigeru SAKAGUCHI ${ }^{\ddagger}$
}

September 26, 2018

\begin{abstract}
A strong comparison principle for semicontinuous viscosity solutions of the prescribed mean curvature equation is considered. The difficulties of the problem come from the fact that this nonlinear equation is non-uniformly elliptic, does not depend on the value of unknown functions, depends on spatial variables and solutions are semicontinuous. Our simple proof of the strong comparison principle consists only of three ingredients, the definition of viscosity solutions, the inf and sup convolutions of functions, and the theory of classical solutions of quasilinear elliptic equations. Once we have the strong comparison principle, we can prove a weak comparison principle for semicontinuous viscosity solutions of the prescribed mean curvature equation in a bounded domain.
\end{abstract}

Key words. Prescribed mean curvature equation, strong comparison principle, semicontinuous viscosity solution.

AMS subject classifications. Primary 35J93 ; Secondary 35D40, 35B50, 35B51

*This research was partially supported by the Grants-in-Aid for Challenging Exploratory Research ( $\sharp$ $25610024)$ and for Scientific Research (B) ( $\sharp 26287020)$ of Japan Society for the Promotion of Science.

${ }^{\dagger}$ Department of Mathematical Sciences, Graduate School of Science and Technology, Tokushima University, Tokushima, 770-8502, Japan. (ohnuma@tokushima-u.ac.jp).

${ }^{\ddagger}$ Research Center for Pure and Applied Mathematics, Graduate School of Information Sciences, Tohoku University, Sendai, 980-8579, Japan. (sigersak@tohoku.ac.jp). 


\section{Introduction}

We consider the prescribed mean curvature equation of the form

$$
\operatorname{div}\left(\frac{D u}{\sqrt{1+|D u|^{2}}}\right)=N H \quad \text { in } \Omega,
$$

where $\Omega$ is a domain in $\mathbf{R}^{N}$ and $N \geq 2$. The function $u: \Omega \rightarrow \mathbf{R}$ is unknown, $D u$ denotes the gradient of $u$ in spatial variables $x$ and $H: \Omega \rightarrow \mathbf{R}$ is a locally Lipschitz continuous function in $\Omega$. When the solution $u$ is Lipschitz continuous, equation (1.1) is regarded as uniformly elliptic. However, when $u$ is only semicontinuous, equation (1.1) can be non-uniformly elliptic.

Our goal is to prove a strong comparison principle for semicontinuous viscosity solutions of the prescribed mean curvature equation. Here, our strong comparison principle is stated as follows: if a lower semicontinuous viscosity supersolution $u$ and an upper semicontinuous viscosity subsolution $v$ satisfy that $u \geq v$ in $\Omega$ and $u\left(x_{0}\right)=v\left(x_{0}\right)$ at some point $x_{0} \in \Omega$, then $u \equiv v$ in $\Omega$.

It is well known that for linear elliptic equations the strong comparison principle is equivalent to the strong maximum principle since the difference of two solutions is still a solution. Here, the strong maximum principle is the following: if a subsolution $u$ satisfies that $u \leq m$ with some constant $m$ and $u\left(x_{0}\right)=m$ at some point $x_{0} \in \Omega$, then $u \equiv m$ in $\Omega$. Evidently the strong comparison principle implies the strong maximum principle provided that the constant $m$ is a supersolution. The strong maximum principle for classical solutions of linear and nonlinear elliptic equations has been well studied (cf. [GT], [PW]). In a book [PS, Theorem 2.1.3 (Tangency Principle), p. 16] we can find the strong comparison principle for classical solutions of nonlinear elliptic equations.

There are some results on the strong maximum principle for weak solutions in the viscosity sense. For notations of viscosity solutions we refer to the literature [CIL] and [Ko]. The strong maximum principle for semicontinuous viscosity solutions has been proved by $[\mathrm{KaKu}, \mathrm{BD},[\mathrm{BB}], \mathrm{KoKo}$, and $[\mathrm{BGI}]$. There are a few papers on the strong comparison principle. Trudinger [T] proved the strong comparison principle for Lipschitz continuous viscosity solutions of uniformly elliptic equations. Ishii and Yoshimura [Y] proved the strong comparison principle for semicontinuous viscosity solutions of uniformly elliptic equations. At the same time Giga and the first author [GO] dealt with the strong comparison principle for semicontinuous viscosity solutions of nonlinear elliptic equations. We recently noticed that the argument in [GO, Proof of Theorem 3.1, pp. 177-179] works 
for uniformly elliptic equations of the form $F\left(D^{2} u\right)=0$, but it does not work for nonuniformly elliptic equations of the form $F\left(D u, D^{2} u\right)=f(x)$ such as (1.1).

In the present paper we consider lower semicontinuous viscosity supersolutions and upper semicontinuous viscosity subsolutions of (1.1). Therefore, we have to deal with nonuniformly elliptic equations. Our proof is different from usual one. After being reduced to the case where both the supersolution $u$ and the subsolution $v$ are bounded, by virtue of Jensen, Lions and Souganidis [JLS], we introduce the inf and sup convolutions of $u$ and $v$ respectively, where those convolutions are continuous functions and moreover they are monotone with respect to the parameter. Then we consider the Dirichlet problems for (1.1) in every sufficiently small ball centered at a point $x_{0}$, where $u$ touches $v$. We choose the continuous boundary data as the inf and sup convolutions of $u$ and $v$, respectively. Since $H$ is locally Lipschitz continuous, by the theory of quasilinear elliptic equations (see [GT]), the gradient estimates of classical solutions are available and these problems have unique classical solutions provided that the ball is sufficiently small. Here the strong comparison principle is applicable to these two classical solutions and also a weak comparison principle is applicable to compare $u$ and $v$ with these two classical solutions, respectively. Eventually, these comparisons yield that $u$ and $v$ coincide with each other on the boundary of each small ball centered at a point $x_{0}$, and hence $u$ and $v$ coincide with each other in a small ball centered at a point $x_{0}$. Then the conclusion follows from the connectedness of the domain.

The present paper is organized as follows. In section 2 we state our main theorem and prove it. In section 3 we give a weak comparison principle as a corollary of our strong comparison principle. In Appendix we prove a weak comparison principle for (1.1) which compares a lower semicontinuous viscosity supersolution with a classical solution, or an upper semicontinuous viscosity subsolution with a classical solution.

\section{Main theorem}

Let $\Omega$ be a domain in $\mathbf{R}^{N}, N \geq 2$ and let $u: \Omega \rightarrow \mathbf{R}$. For functions $u$ we set

$$
M(u):=\operatorname{div}\left(\frac{D u}{\sqrt{1+|D u|^{2}}}\right) .
$$

Here $D u$ denotes the gradient of $u$ in spatial variables $x$. Let $H: \Omega \rightarrow \mathbf{R}$ be a locally Lipschitz continuous function in $\Omega$. Then equation (1.1) is written as

$$
M(u)=N H \quad \text { in } \Omega .
$$


Our main theorem concerns an extension of the strong comparison theorem to semicontinuous viscosity supersolutions and subsolutions of (2.1). We will use the following notations:

$$
\begin{aligned}
\operatorname{USC}(\Omega) & =\{\text { upper semicontinuous functions } u: \Omega \rightarrow \mathbf{R}\}, \\
\operatorname{LSC}(\Omega) & =\{\text { lower semicontinuous functions } u: \Omega \rightarrow \mathbf{R}\} .
\end{aligned}
$$

Also, $\operatorname{USC}(\bar{\Omega}), \operatorname{LSC}(\bar{\Omega})$ are defined similarly.

Theorem 2.1 Let $u \in \operatorname{LSC}(\Omega)$ be a viscosity supersolution of (2.1), that is,

$$
M(u) \leq N H \quad \text { in } \Omega
$$

in the viscosity sense, and let $v \in \operatorname{USC}(\Omega)$ be a viscosity subsolution of (2.1), that is,

$$
M(v) \geq N H \quad \text { in } \Omega
$$

in the viscosity sense. Assume that $u \geq v$ in $\Omega$ and that $u\left(x_{0}\right)=v\left(x_{0}\right)$ at some point $x_{0} \in \Omega$. Then $u \equiv v$ in $\Omega$.

Remark $2.2 A$ continuous viscosity solution $u$ of (2.1) means that $u \in C(\Omega)$ is both a viscosity supersolution and subsolution of (2.1). Combining the results of $[\mathrm{B}]$ and $[\mathrm{T}]$ yields the strong comparison principle for continuous viscosity solutions of (2.1). Indeed, it is shown in [B] that continuous viscosity solutions of (2.1) are Lipschitz continuous. Then, equation (2.1) is regarded as uniformly elliptic, and hence thanks to Trudinger's results in [T] we see that the strong comparison principle for continuous viscosity solutions of (2.1) holds.

The following weak comparison principle, which is proved in more general form in $\mathrm{KaKu}$, Theorem 3, p. 475], plays a key role in the present paper. Therefore we give a simple proof directly by using the implicit function theorem and the definition of viscosity solutions in the Appendix.

Proposition 2.3 Let $\Omega$ be a bounded domain in $\mathbf{R}^{N}$. Let $u \in \operatorname{LSC}(\bar{\Omega})$ be a viscosity supersolution of (2.1) and let $v \in C^{2}(\Omega) \cap C(\bar{\Omega})$ be a classical solution of (2.1). Assume that $u \geq v$ on $\partial \Omega$, then $u \geq v$ in $\Omega$. Similarly, it holds for a classical solution $u \in$ $C^{2}(\Omega) \cap C(\bar{\Omega})$ and a viscosity subsolution $v \in \operatorname{USC}(\bar{\Omega})$ of (2.1).

Now we are in a position to prove Theorem 2.1. 
Proof of Theorem 2.1.

1st step: Reduction to the case where both $u$ and $v$ are bounded: Let $E$ be a bounded domain with $\bar{E} \subset \Omega$ and $x_{0} \in E$. Since $u$ is lower semicontinuous and $v$ is upper semicontinuous, there exists $K>0$ such that

$$
u>-K \text { and } v<K \text { in } \bar{E} \text {. }
$$

We use a notation $B(x, r)$ as an open ball in $\mathbf{R}^{N}$ of radius $r>0$ centered at $x \in \mathbf{R}^{N}$. For simplicity we write in particular $B_{r}:=B\left(x_{0}, r\right)$ for every $r>0$. Choose a positive number $R>0$ satisfying

$$
-\frac{1}{R} \leq H \leq \frac{1}{R} \quad \text { in } \bar{E} \quad \text { and } \overline{B_{R}} \subset E .
$$

For $x \in \overline{B_{R}}$ we set

$$
\begin{aligned}
& u_{R}(x):=\min \left\{u(x), K+\sqrt{R^{2}-\left|x-x_{0}\right|^{2}}\right\}, \\
& v_{R}(x):=\max \left\{v(x),-K-\sqrt{R^{2}-\left|x-x_{0}\right|^{2}}\right\} .
\end{aligned}
$$

Then $u_{R} \in \operatorname{LSC}\left(\overline{B_{R}}\right)$ and $v_{R} \in \operatorname{USC}\left(\overline{B_{R}}\right)$ are bounded in $\overline{B_{R}}$. Moreover, $u_{R}$ is a viscosity supersolution of (2.1) in $B_{R}$ and $v_{R}$ is a viscosity subsolution of (2.1) in $B_{R}$.

Since $v \leq u$ in $\Omega$ and $v\left(x_{0}\right)=u\left(x_{0}\right)$, we have $v \leq v_{R} \leq u_{R} \leq u$ in $\overline{B_{R}}$ and $v_{R}\left(x_{0}\right)=$ $u_{R}\left(x_{0}\right)$. By the definition of $u_{R}$ and $v_{R}$ we see that if $u_{R} \equiv v_{R}$ in $\overline{B_{R}}$, then $u \equiv v$ in $\overline{B_{R}}$. Thus we may assume that $u$ and $v$ are bounded in $\overline{B_{R}}$.

\section{2nd step: Introducing the inf and sup convolutions of the super and subso-}

lutions: We introduce the inf and sup convolutions of $u$ and $v$, respectively, as in [JLS, Proof of Proposition 2, p. 977]. For small $\varepsilon>0$, we set

$$
\begin{array}{ll}
u_{\varepsilon}(x):=\inf _{y \in \overline{B_{R}}}\left\{u(y)+\frac{|x-y|^{2}}{2 \varepsilon}\right\} & \text { for } x \in \overline{B_{R}}, \\
v^{\varepsilon}(x):=\sup _{y \in \overline{B_{R}}}\left\{v(y)-\frac{|x-y|^{2}}{2 \varepsilon}\right\} & \text { for } x \in \overline{B_{R}} .
\end{array}
$$

Notice that $u_{\varepsilon}, v^{\varepsilon} \in C\left(\overline{B_{R}}\right)$, and at each point $x \in \overline{B_{R}}$ the inf convolution $u_{\varepsilon}(x)$ increases to $u(x)$ and the sup convolution $v^{\varepsilon}(x)$ decreases to $v(x)$ as $\varepsilon$ decreases to 0 .

Proposition 2.4 For each $\varepsilon>0, v^{\varepsilon} \geq u_{\varepsilon}$ in $\overline{B_{\frac{R}{2}}}$.

Proof. By setting $\rho=\frac{R}{2}$, we observe that for every $0<r \leq \rho$

$$
\frac{1}{r} \geq \frac{N}{N-1}|H| \text { in } \partial B_{r} \text { and } \int_{B_{r}}|H|^{N} d x<\omega_{N},
$$

where $\omega_{N}$ denotes the Lebesgue measure of the unit ball in $\mathbf{R}^{N}$. 
Fix $\varepsilon_{0}>0$ arbitrarily. Let us show that

$$
v^{\varepsilon_{0}} \geq u_{\varepsilon_{0}} \quad \text { in } \overline{B_{\rho}}
$$

For each $\varepsilon \in\left(0, \varepsilon_{0}\right]$, we set

$$
\delta_{\varepsilon}:=\min _{y \in \overline{B_{\rho}}}\left(u_{\varepsilon}(y)-v^{\varepsilon}(y)\right) .
$$

Since $u_{\varepsilon}-v^{\varepsilon}$ is continuous in $\overline{B_{R}}, \delta_{\varepsilon}$ is well defined. By observing that

$$
\min _{y \in \overline{B_{\rho}}}\left(u_{\varepsilon}(y)-v^{\varepsilon}(y)\right) \leq u_{\varepsilon}\left(x_{0}\right)-v^{\varepsilon}\left(x_{0}\right) \leq u\left(x_{0}\right)-v\left(x_{0}\right)=0,
$$

we know $\delta_{\varepsilon} \leq 0$. Since $u_{\varepsilon}(x)$ increases to $u(x)$ and $v^{\varepsilon}(x)$ decreases to $v(x)$ as $\varepsilon$ decreases to 0 at each $x \in \overline{B_{R}}, \delta_{\varepsilon}$ is monotone increasing as $\varepsilon$ decreases to 0 . Let us show a lemma.

\section{Lemma 2.5}

$$
\lim _{\varepsilon \rightarrow 0} \delta_{\varepsilon}=0
$$

Proof. We may set

$$
\lim _{\varepsilon \rightarrow 0} \delta_{\varepsilon}=-\lambda
$$

for some number $\lambda \geq 0$. For each $\varepsilon>0$ there exists a point $y_{\varepsilon} \in \overline{B_{\rho}}$ such that $\delta_{\varepsilon}=$ $u_{\varepsilon}\left(y_{\varepsilon}\right)-v^{\varepsilon}\left(y_{\varepsilon}\right)$ and moreover there exist points $y_{1, \varepsilon}, y_{2, \varepsilon} \in \overline{B_{R}}$ such that

$$
\begin{aligned}
& u_{\varepsilon}\left(y_{\varepsilon}\right):=u\left(y_{1, \varepsilon}\right)+\frac{\left|y_{\varepsilon}-y_{1, \varepsilon}\right|^{2}}{2 \varepsilon}, \\
& v^{\varepsilon}\left(y_{\varepsilon}\right):=v\left(y_{2, \varepsilon}\right)-\frac{\left|y_{\varepsilon}-y_{2, \varepsilon}\right|^{2}}{2 \varepsilon} .
\end{aligned}
$$

Since $u_{\varepsilon}\left(y_{\varepsilon}\right), v^{\varepsilon}\left(y_{\varepsilon}\right), u\left(y_{1, \varepsilon}\right)$ and $v\left(y_{2, \varepsilon}\right)$ are bounded, we must have

$$
y_{\varepsilon}-y_{1, \varepsilon} \rightarrow 0 \text { and } \quad y_{\varepsilon}-y_{2, \varepsilon} \rightarrow 0 \quad \text { as } \varepsilon \rightarrow 0 .
$$

On the other hand, the Bolzano-Weierstrass theorem yields that there exist a sequence $\left\{\varepsilon_{j}\right\}$ which decreases to 0 as $j \rightarrow \infty$ and $x_{*} \in \overline{B_{\rho}}$ satisfying

$$
y_{\varepsilon_{j}} \rightarrow x_{*} \quad \text { as } j \rightarrow \infty .
$$

Then it follows from (2.3) and (2.4) that

$$
y_{1, \varepsilon_{j}}, y_{2, \varepsilon_{j}} \rightarrow x_{*} \quad \text { as } j \rightarrow \infty .
$$

Since $\frac{\left|y_{\varepsilon_{j}}-y_{1, \varepsilon_{j}}\right|^{2}}{2 \varepsilon_{j}}$ and $\frac{\left|y_{\varepsilon_{j}}-y_{2, \varepsilon_{j}}\right|^{2}}{2 \varepsilon_{j}}$ are bounded, by taking a subsequence if necessary, we may suppose that

$$
\frac{\left|y_{\varepsilon_{j}}-y_{1, \varepsilon_{j}}\right|^{2}}{2 \varepsilon_{j}} \rightarrow \beta_{1}(\geq 0) \quad \text { and } \quad \frac{\left|y_{\varepsilon_{j}}-y_{2, \varepsilon_{j}}\right|^{2}}{2 \varepsilon_{j}} \rightarrow \beta_{2}(\geq 0) \quad \text { as } \quad j \rightarrow \infty
$$


for some numbers $\beta_{1}, \beta_{2}$. The lower semicontinuity of $u$ and $-v$ at $x_{*}$ yields that, for every $\eta>0$, there exists $\gamma>0$ such that if $\left|x-x_{*}\right|<\gamma$ then

$$
u(x)>u\left(x_{*}\right)-\eta \quad \text { and } \quad-v(x)>-v\left(x_{*}\right)-\eta .
$$

By (2.5) there exists $n_{0} \in \mathbf{N}$ such that if $j \geq n_{0}$ then

$$
u\left(y_{1, \varepsilon_{j}}\right)>u\left(x_{*}\right)-\eta \text { and }-v\left(y_{2, \varepsilon_{j}}\right)>-v\left(x_{*}\right)-\eta .
$$

Hence, for $j \geq n_{0}$

$$
\begin{aligned}
\delta_{\varepsilon_{j}} & =u_{\varepsilon_{j}}\left(y_{\varepsilon_{j}}\right)-v^{\varepsilon_{j}}\left(y_{\varepsilon_{j}}\right)=u\left(y_{1, \varepsilon_{j}}\right)+\frac{\left|y_{\varepsilon_{j}}-y_{1, \varepsilon_{j}}\right|^{2}}{2 \varepsilon_{j}}-v\left(y_{2, \varepsilon_{j}}\right)+\frac{\left|y_{\varepsilon_{j}}-y_{2, \varepsilon_{j}}\right|^{2}}{2 \varepsilon_{j}} \\
& >u\left(x_{*}\right)-v\left(x_{*}\right)-2 \eta+\frac{\left|y_{\varepsilon_{j}}-y_{1, \varepsilon_{j}}\right|^{2}}{2 \varepsilon_{j}}+\frac{\left|y_{\varepsilon_{j}}-y_{2, \varepsilon_{j}}\right|^{2}}{2 \varepsilon_{j}} .
\end{aligned}
$$

Letting $j \rightarrow \infty$ yields that for every $\eta>0$

$$
0 \geq-\lambda \geq u\left(x_{*}\right)-v\left(x_{*}\right)-2 \eta+\beta_{1}+\beta_{2} .
$$

Since $u\left(x_{*}\right) \geq v\left(x_{*}\right), \beta_{1} \geq 0$ and $\beta_{2} \geq 0$, we see that $0 \leq \lambda \leq 2 \eta$ and $0 \leq \beta_{1}+\beta_{2} \leq 2 \eta$ for every $\eta>0$. Thus we conclude that $\lambda=\beta_{1}=\beta_{2}=0$ and the proof of Lemma 2.5 is finished.

We return to the proof of Proposition 2.4. In order to prove that

$$
v^{\varepsilon_{0}} \geq u_{\varepsilon_{0}} \text { in } \overline{B_{\rho}},
$$

let us show that for each $r$ with $0<r \leq \rho$,

$$
v^{\varepsilon_{0}}(x) \geq u_{\varepsilon_{0}}(x) \text { for all } x \in \partial B_{r}
$$

Suppose that there exist $r$ with $0<r \leq \rho$ and a point $x_{1} \in \partial B_{r}$ so that $u_{\varepsilon_{0}}\left(x_{1}\right)-$ $v^{\varepsilon_{0}}\left(x_{1}\right)>0$. Since $u_{\varepsilon_{0}}(x)-v^{\varepsilon_{0}}(x)$ is continuous, there exist $r_{1}$ with $0<r_{1}<\frac{r}{4}$ and $\beta>0$ such that

$$
u_{\varepsilon_{0}}(x)-v^{\varepsilon_{0}}(x) \geq \beta \text { in } \overline{B\left(x_{1}, r_{1}\right)}
$$

We divide $\partial B_{r}$ into two pieces:

$$
\Gamma_{+}:=\partial B_{r} \cap \overline{B\left(x_{1}, r_{1}\right)} \text { and } \Gamma_{0}:=\partial B_{r} \backslash \Gamma_{+} .
$$

Clearly $\partial B_{r}$ is a disjoint union of $\Gamma_{+}$and $\Gamma_{0}$.

Note that (2.6) gives in particular

$$
u_{\varepsilon_{0}}(x) \geq v^{\varepsilon_{0}}(x)+\beta \quad \text { on } \Gamma_{+} .
$$


Then for every $0<\varepsilon \leq \varepsilon_{0}$, by the monotonicity of $u_{\varepsilon}$ and $v^{\varepsilon}$

$$
u_{\varepsilon}(x) \geq u_{\varepsilon_{0}}(x) \geq v^{\varepsilon_{0}}(x)+\beta \geq v^{\varepsilon}(x)+\beta \quad \text { on } \Gamma_{+},
$$

and by the definition of $\delta_{\varepsilon}$ we see that

$$
u_{\varepsilon}(x) \geq v^{\varepsilon}(x)+\delta_{\varepsilon} \quad \text { on } \partial B_{r}
$$

Since $H$ is Lipschitz continuous in $\overline{B_{r}}$, we see that the interior estimates of $G T$, Corollary 16.7, p. 407] are available. Therefore it follows from (2.2) and the theory of the prescribed mean curvature equation ([GT, Theorem 16.10, p. 408$])$ that there exist $\hat{u}_{\varepsilon}, \hat{v}^{\varepsilon} \in C^{2}\left(B_{r}\right) \cap C\left(\overline{B_{r}}\right)$ satisfying

$$
\begin{array}{ll}
M\left(\hat{u}_{\varepsilon}\right)=M\left(\hat{v}^{\varepsilon}\right)=N H & \text { in } B_{r}, \\
\hat{u}_{\varepsilon}=u_{\varepsilon} \quad \text { and } \quad \hat{v}^{\varepsilon}=v^{\varepsilon} & \text { on } \partial B_{r} .
\end{array}
$$

Notice that $M\left(\hat{v}^{\varepsilon}+\delta_{\varepsilon}\right)=N H$ in $B_{r}$. By Proposition 2.3 and (2.8) we observe that

$$
v+\delta_{\varepsilon} \leq \hat{v}^{\varepsilon}+\delta_{\varepsilon} \leq \hat{u}_{\varepsilon} \leq u \quad \text { in } \overline{B_{r}} .
$$

Also, $\hat{u}_{\varepsilon}$ increases and $\hat{v}^{\varepsilon}$ decreases as $\varepsilon$ decreases to 0 by the monotonicity of $u_{\varepsilon}$ and $v^{\varepsilon}$.

The boundedness of $\left\{\hat{u}_{\varepsilon}\right\}$ and $\left\{\hat{v}^{\varepsilon}\right\}$ together with the interior estimates of [GT, Corollary 16.7, p. 407] yields that there exist $\hat{u}_{0}, \hat{v}^{0} \in C^{2}\left(B_{r}\right)$ such that

$$
\begin{aligned}
& \hat{u}_{\varepsilon} \rightarrow \hat{u}_{0} \text { and } \hat{v}^{\varepsilon} \rightarrow \hat{v}^{0} \text { as } \varepsilon \rightarrow 0 \text { uniformly on compact sets in } B_{r}, \\
& M\left(\hat{u}_{0}\right)=M\left(\hat{v}^{0}\right)=N H \text { in } B_{r} .
\end{aligned}
$$

Therefore, since $\lim _{\varepsilon \rightarrow 0} \delta_{\varepsilon}=0$, we observe from (2.9) that

$$
v \leq \hat{v}^{0} \leq \hat{u}_{0} \leq u \quad \text { in } \quad B_{r} .
$$

Since $v\left(x_{0}\right)=u\left(x_{0}\right)$, we have that $\hat{v}^{0}\left(x_{0}\right)=\hat{u}_{0}\left(x_{0}\right)$. By the strong comparison principle for classical solutions we see that

$$
\hat{v}^{0}(x) \equiv \hat{u}_{0}(x) \quad \text { in } B_{r}
$$

However, by (2.7)

$$
\hat{u}_{\varepsilon_{0}}(x) \geq \hat{v}^{\varepsilon_{0}}(x)+\beta \quad \text { on } \Gamma_{+} .
$$

Hence it follows from the continuity of $\hat{u}_{\varepsilon_{0}}$ and $\hat{v}^{\varepsilon_{0}}$ that there exist $\beta_{3}$ with $0<\beta_{3} \leq \beta$ and $r_{2}$ with $0<r_{2} \leq r_{1}$ satisfying

$$
\hat{u}_{\varepsilon_{0}}(x) \geq \hat{v}^{\varepsilon_{0}}(x)+\beta_{3} \quad \text { in } \quad B_{r} \cap \overline{B\left(x_{1}, r_{2}\right)} .
$$


By the monotonicity of $u_{\varepsilon}$ and $v^{\varepsilon}$ for $0<\varepsilon \leq \varepsilon_{0}$ we observe that

$$
\hat{u}_{\varepsilon}(x) \geq \hat{u}_{\varepsilon_{0}}(x) \geq \hat{v}^{\varepsilon_{0}}(x)+\beta_{3} \geq \hat{v}^{\varepsilon}(x)+\beta_{3} \quad \text { in } \quad B_{r} \cap \overline{B\left(x_{1}, r_{2}\right)} .
$$

Therefore letting $\varepsilon \rightarrow 0$ yields that

$$
\hat{u}_{0}(x) \geq \hat{v}^{0}(x)+\beta_{3} \quad \text { in } \quad B_{r} \cap \overline{B\left(x_{1}, r_{2}\right)} .
$$

This contradicts (2.10) .

Eventually, we conclude that $v^{\varepsilon_{0}} \geq u_{\varepsilon_{0}}$ on $\partial B_{r}$. Since this holds for every $0<r \leq \rho$, we have

$$
v^{\varepsilon_{0}} \geq u_{\varepsilon_{0}} \quad \text { in } \overline{B_{\rho}}
$$

3rd step: Completion of the proof of Theorem 2.1 By Proposition 2.4, letting $\varepsilon \rightarrow 0$ yields that

$$
v \geq u \quad \text { in } \overline{B_{\rho}},
$$

which shows that $u \equiv v$ in $\overline{B_{\rho}}$. Since $\Omega$ is connected, we conclude that $u \equiv v$ in $\Omega$.

\section{A weak comparison principle for semicontinuous viscosity solutions of the prescribed mean curvature equation}

The strong comparison principle proved in section 2 yields a weak comparison principle for semicontinuous viscosity solutions of the prescribed mean curvature equation in a bounded domain.

Theorem 3.1 Let $\Omega$ be a bounded domain in $\mathbf{R}^{n}$. Let $u \in \operatorname{LSC}(\bar{\Omega})$ and $v \in \operatorname{USC}(\bar{\Omega})$ be viscosity super and subsolutions of (2.1), respectively. Assume that $u \geq v$ on $\partial \Omega$. Then $u \geq v$ in $\Omega$, and hence either $u \equiv v$ in $\Omega$ or $u>v$ in $\Omega$.

Proof. Suppose that there exists a point $x_{1} \in \bar{\Omega}$ satisfying

$$
\theta:=\min _{x \in \bar{\Omega}}(u-v)(x)=(u-v)\left(x_{1}\right)<0 .
$$

Hence $x_{1} \in \Omega$, since $u \geq v$ on $\partial \Omega$. Then we observe that

$$
u \geq v+\theta \quad \text { in } \Omega \text { and } \quad u\left(x_{1}\right)=v\left(x_{1}\right)+\theta .
$$

Note that $v+\theta$ is also a viscosity subsolution of (2.1). By Theorem 2.1 we have that $u \equiv v+\theta$ in $\Omega$, which contradicts the assumption that $u \geq v$ on $\partial \Omega$. Therefore we see that $u \geq v$ in $\Omega$.

Moreover, if there exists a point $x_{2} \in \Omega$ so that $u\left(x_{2}\right)=v\left(x_{2}\right)$, then $u \equiv v$ in $\Omega$ by Theorem 2.1, which concludes that either $u \equiv v$ in $\Omega$ or $u>v$ in $\Omega$. 


\section{Appendix}

Although Proposition 2.3 was already proved in [KaKu, Theorem 3, p. 457], for convenience we will give a simple proof directly by using the implicit function theorem and the definition of viscosity solutions.

Proof of Proposition 2.3. By the argument in [MS, Theorem A.1, p. 253], which applies Sard's theorem to a smooth function being comparable to the distance function to the closed set $\mathbb{R}^{N} \backslash \Omega$ due to Calderón and Zygmund [Z, Lemma 3.6.1, p. 136] (see also [CZ, Lemma 3.2, p. 185]), we observe that for each small $\varepsilon>0$ there exists a smooth open set $\Omega_{\varepsilon} \subset \subset \Omega$ with $\Omega_{\varepsilon^{\prime}} \subset \Omega_{\varepsilon}$ if $\varepsilon<\varepsilon^{\prime}$ and $\Omega_{\varepsilon} \rightarrow \Omega$ as $\varepsilon \rightarrow 0$. Since $\Omega$ is bounded, we notice that $\Omega_{\varepsilon}$ is a union of a finite number of smooth domains.

Let $u \in \operatorname{LSC}(\bar{\Omega})$ be a viscosity supersolution of (2.1) and let $v \in C^{2}(\Omega) \cap C(\bar{\Omega})$ be a classical solution of (2.1). Assume that $u \geq v$ on $\partial \Omega$. Since $H$ is locally Lipschitz continuous, we see that the interior estimates of [GT, Corollary 16.7, p. 407] are available. Therefore, with the aid of the Schauder interior estimates for elliptic equations, there exists a number $\alpha$ with $0<\alpha<1$ depending on $\varepsilon$ such that $v \in C^{2, \alpha}\left(\overline{\Omega_{\varepsilon}}\right)$. We can write

$$
\Omega_{\varepsilon}=\bigcup_{j=1}^{n(\varepsilon)} D_{\varepsilon, j} .
$$

Consider an arbitrary $D_{\varepsilon, j}$. For simplicity we will write $D$ instead of $D_{\varepsilon, j}$. Note that $\partial D$ is close to $\partial \Omega$ if $\varepsilon>0$ is sufficiently small. Since $u \in \operatorname{LSC}(\bar{\Omega}), v \in C(\bar{\Omega})$ and $u \geq v$ on $\partial \Omega$, there exists $\tau(\varepsilon)>0$ satisfying $\lim _{\varepsilon \rightarrow 0} \tau(\varepsilon)=0$ and $u>v-\tau(\varepsilon)$ on $\partial D$.

Set $w_{\varepsilon}:=v-\tau(\varepsilon)$ and we have

$$
M\left(w_{\varepsilon}\right)=N H \quad \text { in } D .
$$

We set

$$
\begin{aligned}
& X \quad:=\left\{f \in C^{2, \alpha}(\bar{D}) \mid f=0 \text { on } \partial D\right\}, \\
& F \quad: X \times \mathbf{R} \ni(f, s) \mapsto M\left(w_{\varepsilon}+f\right)-N(H+s) \in C^{\alpha}(\bar{D}) .
\end{aligned}
$$

We use the implicit function theorem for $X$ and $F$ ( see [AP, Theorem 2.3, p. 38], [D, Theorem 15.1, p. 148] for instance ). For each $0<\delta<<1$ there exists $\tilde{w}_{\varepsilon, \delta} \in C^{2, \alpha}(\bar{D})$ satisfying

$$
\begin{aligned}
& M\left(\tilde{w}_{\varepsilon, \delta}\right)=N(H+\delta) \text { in } D, \\
& \tilde{w}_{\varepsilon, \delta}=w_{\varepsilon} \quad \text { on } \partial D .
\end{aligned}
$$

Note that $\tilde{w}_{\varepsilon, 0}=w_{\varepsilon}$. Then we have

$$
u \geq \tilde{w}_{\varepsilon, \delta} \quad \text { in } \bar{D} .
$$


Indeed, suppose that there exists a point $z \in \bar{D}$ satisfying

$$
\min _{x \in \bar{D}}\left(u-\tilde{w}_{\varepsilon, \delta}\right)(x)=\left(u-\tilde{w}_{\varepsilon, \delta}\right)(z)<0 .
$$

Hence $z \in D$, since $u-\tilde{w}_{\varepsilon, \delta}=u-w_{\varepsilon}>0$ on $\partial D$. Moreover, since $u$ is a viscosity supersolution of (2.1), we have from (3.3) that $M\left(\tilde{w}_{\varepsilon, \delta}\right)(z) \leq N H(z)$. This contradicts the fact that $M\left(\tilde{w}_{\varepsilon, \delta}\right)(z)=N(H(z)+\delta)$ with $\delta>0$.

Letting $\delta \rightarrow 0$ in (3.2) yields that

$$
u \geq w_{\varepsilon} \quad \text { in } \bar{D} \text {. }
$$

Hence it follows from (3.1) that

$$
u \geq v-\tau(\varepsilon) \quad \text { in } \Omega_{\varepsilon}
$$

Thus, letting $\varepsilon \rightarrow 0$ yields that $u \geq v$ in $\Omega$, which completes the proof of Proposition 2.3.

\section{Acknowledgements}

The authors are grateful to Professor Hiroyoshi Mitake for informing them of a gap in the proof in [GO] when one deals with the prescribed mean curvature equation.

\section{References}

[AP] A. Ambrosetti and G. Prodi, A Primer of Nonlinear Analysis, Cambridge University Press, 1995.

[B] G. Barles, Interior gradient bounds for mean curvature equation by viscosity solutions methods, Differential Integral Equations, 4, (1991), 263-275.

[BB] G. Barles and J. Busca, Exisence and comparison results for fully nonlinear degenerate elliptic equations without zeroth-order term, Comm. Partial Differential Equations, 26, (2001), 2323-2337.

[BD] M. Bardi and F. Da Lio, On the strong maximum principle for fully nonlinear degenerate elliptic equations, Arch. Math., 73, (1999), 276-285.

[BGI] I. Birindelli, G. Galise and H. Ishii, A family of degenerate elliptic operators: Maximum principle and its consequences, Ann. I. H. Poincaré - AN, 35, (2018), $417-441$. 
[CZ] A. P. Calderón and A. Zygmund, Local properties of solutions of elliptic partial differential equations, Studia Math., 20 (1961), 171-225.

[CIL] M. G. Crandall, H. Ishii and P. L. Lions, User's guide to viscosity solutions of second order partial differential equations, Bull. Amer. Math. Soc., 27, (1992), $1-67$.

[D] K. Deimling, Nonlinear Functional Analysis, Dover Publications, 2010.

[GO] Y. Giga and M. Ohnuma, On strong comparison principle for semicontinuous viscosity solutions of some nonlinear elliptic equations, Int. J. of Pure and Appl. Math., 22, (2005), 165-184.

[GT] D. Gilbarg and N. S. Trudinger, Elliptic Partial Differential Equations of Second Order, Second Edition, Springer-Verlag, 1983.

[IY] H. Ishii and Y. Yoshimura, Demi-eigenvalues for uniformly elliptic Isaccs operators, preprint.

[JLS] R. Jensen, P. L. Lions and P. E. Souganidis, A uniqueness result for viscosity solutions of second order fully nonlinear partial differential equations, Proc. Amer. Math. Soc., 102, (1988), 975-978.

[KaKu] B. Kawohl and N. Kutev, Strong maximum principle for semicontinuous viscosity solutions of nonlinear partial differential equations, Arch. Math., 70, (1998), 470478.

[Ko] S. Koike, Beginner's Guide to the Theory of Viscosity Solutions, MSJ memoir 13, Math. Soc. Japan, 2004.

[KoKo] S. Koike and T. Kosugi Remarks on the comparison principle for quasilinear PDE with no zeroth order terms, Comm. Pure Appl. Anal., 14, (2015), 133-142.

[MS] R. Magnanini and S. Sakaguchi, Interaction between nonlinear diffusion and geometry of domain, J. Differential Equations, 252, (2012), 236-257.

[PS] P. Pucci and J. Serrin, The Strong Maximum Principle, Progress in Nonlinear Differential Equations and Their Applications 73, Birkhauser, 2007.

[PW] M. H. Protter and H. Weinberger, Maximum Principle in Differential Equations, Springer-Verlag, 1984 
[T] N. S. Trudinger, Comparison principle and pointwise estimates for viscosity solutions, Rev. Mat. Iberoamericana, 4, (1988), 453-468.

[Z] W. P. Ziemer, Weakly Differentiable Functions, Graduate Texts in Math. 120, Springer-Verlag, New York, Berlin, Heidelberg, London, Paris, Tokyo, Hong Kong, 1989. 